\title{
The details are not so devilish
}

Devil facial tumour disease (DFTD) is a fatal cancer that affects the Tasmanian devil (Sarcophilus harrisii), an endangered species that is predicted to be extinct in 25-35 years as a result of DFTD. DFTD is thought to be a transmissible allograft tumour of neuroendocrine origin, but little is known about its biology. Therefore, Elizabeth Murchison and colleagues have used genomic and transcriptomic profiling to find out more.

Using samples from 25 matched DFTD tumours and host tissue and 10 unaffected devils the authors found that all tumours had similar genotypes at 14 microsatellite loci and that these tumour genotypes were distinct from the matched host or unaffected tissue samples. This is consistent with previous reports that DFTD tumours are transmissible allografts. Next, they used deep sequencing to isolate microRNAs (miRNAs) from ten different tissues and five DFTD tumours. They identified 114 miRNAs from both the normal tissues and DFTD tumours and these were expressed in a tissue-specific manner. The miRNA expression profile in DFTD tumours was distinct and this was most similar to the miRNA expression profile of brain tissue. Interestingly, some of the miRNAs expressed in the DFTD tumours are associated with cancer in humans.

Next, Murchison and colleagues sequenced the transcriptome from a DFTD tumour and the testis from an affected devil (as the widest range of genes is expressed by the testis) and found that $0.4 \%$ of the genome was differentially expressed between the tumour and the testis. Of the top 20 differentially expressed genes, 9 were associated with myelination, including myelin basic protein $(M B P)$. Components of the regulatory network that controls the differentiation of Schwann cells - which produce myelin in the peripheral nervous system - were also differentially expressed in the DFTD tumour. Furthermore, using immunohistochemistry, the authors showed that the Schwann cell-specific myelin protein periaxin (PRX) was expressed in all cells of 20 DFTD tumours and 10 DFTD metastases but was not expressed by non-DFTD tumours. Therefore, PRX staining could be a diagnostic marker. Collectively - and surprisingly given the undifferentiated state of DFTD tumours - these data indicate that DFTD originates from Schwann cells (which are highly differentiated) and is probably a peripheral nerve sheath tumour. However, it is important to note that Schwann cells and neuroendocrine cells both derive from the neural crest and so DFTD tumours might have arisen from a mutual precursor cell.

Although many questions remain about the biology of DFTD, particularly regarding how these tumours evade the immune system of multiple hosts, these genomic and transcriptomic data will be useful in the efforts to prevent the extinction of the Tasmanian devil.

Gemma K. Alderton

ORIGINAL RESEARCH PAPER Murchison, E. P. et al. The Tasmanian devil transcriptome reveals Schwann cell origins of a clonally transmissable cancer. Science 327, 84-87 (2010) FURTHER READING McAloose, D. \& Newton, A. L. Wildlife cancer: a conservation perspective. Nature Rev. Cancer 9, 517-526 (2009) 